\title{
Striking the Right Balance: Evidence to Inform Combined Physical Activity and Sedentary Behavior Recommendations
}

\section{Sebastien F.M. Chastin, Duncan E. McGregor, Stuart J.H. Biddle, Greet Cardon, Jean-Philippe Chaput, Philippa M. Dall, Paddy C. Dempsey, Loretta DiPietro, Ulf Ekelund, Peter T. Katzmarzyk, Michael Leitzmann, Emmanuel Stamatakis, and Hidde P. Van der Ploeg}

\begin{abstract}
Background: Crucial evidence gaps regarding: (1) the joint association of physical activity and sedentary time with health outcomes and (2) the benefits of light-intensity physical activity were identified during the development of recommendations for the World Health Organization Guidelines on physical activity and sedentary behavior (SB). The authors present alternative ways to evidence the relationship between health outcomes and time spent in physical activity and SB and examine how this could be translated into a combined recommendation in future guidelines. Methods: We used compositional data analysis to quantify the dose-response associations between the balance of time spent in physical activity and SB with all-cause mortality. The authors applied this approach using 2005-2006 National Health and Nutrition Examination Survey accelerometer data. Results: Different combinations of time spent in moderate- to vigorous-intensity physical activity, light-intensity physical activity, and SB are associated with similar all-cause mortality risk level. A balance of more than 2.5 minutes of moderate- to vigorous-intensity physical activity per hour of daily sedentary time is associated with the same magnitude of risk reduction for all-cause mortality as obtained by being physically active according to the current recommendations. Conclusion: This method could be applied to provide evidence for more flexible recommendations in the future with options to act on different behaviors depending on individuals' circumstances and capacity.
\end{abstract}

Keywords: 24-hour, sitting, exercise, guidelines, public health

(C) 2021 The Authors. Published by Human Kinetics, Inc. This is an Open Access article distributed under the terms of the Creative Commons Attribution-NonCommercial-NoDerivatives 4.0 International License, CC BY-NC-ND 4.0, which permits the copy and redistribution in any medium or format, provided it is not used for commercial purposes, no modifications are made, appropriate credit is given, and a link to the license is provided. See http://creativecommons.org/licenses/by-nc-nd/4.0. This license does not cover any third-party material that may appear with permission in the article. For commercial use, permission should be requested from Human Kinetics, Inc., through the Copyright Clearance Center (http://www.copyright.com).

Chastin, McGregor, and Dall are with the School of Health and Life Science, Glasgow Caledonian University, Glasgow, United Kingdom. Chastin and Cardon are with the Department of Sport and Life Science, Ghent University, Ghent, Belgium. McGregor is with Biomathematics and Statistics Scotland, Edinburgh, United Kingdom. Biddle is with the Centre for Health Research, University of Southern Queensland, Springfield, QLD, Australia. Chaput is with the Healthy Active Living and Obesity Research Group, CHEO Research Institute, Ottawa, ON, Canada. Dempsey is with the MRC Epidemiology Unit, Institute of Metabolic Science, University of Cambridge, Cambridge Biomedical Campus, Cambridge, United Kingdom; the Baker Heart and Diabetes Institute, Melbourne, VIC, Australia; and the Diabetes Research Centre, University of Leicester, Leicester General Hospital, Leicester, United Kingdom. DiPietro is with the Milken Institute School of Public Health, The George Washington University, Washington, DC, USA. Ekelund is with the Department of Sport Medicine, Norwegian School of Sport Sciences, Oslo, Norway; and the Department for Chronic Diseases and Ageing, Norwegian Institute of Public Health, Oslo, Norway. Katzmarzyk is with the Pennington Biomedical Research Center, Baton Rouge, LA, USA. Leitzmann is with the Department of Epidemiology and Preventive Medicine, University of Regensburg, Germany. Stamatakis is with the Charles Perkins Centre, School of Health Sciences, Faculty of Medicine and Health, University of Sydney, Sydney, NSW, Australia. van der Ploeg is with the Department of Public and Occupational Health, Amsterdam Public Health Research Institute, Amsterdam UMC, Vrije Universiteit Amsterdam, The Netherlands; and the Sydney School of Public Health, The University of Sydney, Sydney, NSW, Australia. Chastin (sebastien.chastin@ gcu.ac.uk) is corresponding author.
In 2019, the World Health Organization (WHO) convened a Guidelines Development Group including public health scientists and practitioners to contribute to the development of the 2020 Guidelines on Physical Activity and Sedentary Behaviour. ${ }^{1}$ The Guidelines Development Group reviewed the current scientific evidence, building on the work completed for recent guidelines internationally ${ }^{2-11}$ about the relationship between physical activity, sedentary behavior (SB), and health. The end goal was to provide recommendations on the amount of physical activity and SB associated with favorable health outcomes in children and adolescents, adults, older adults ( $>65 \mathrm{y}$ ), as well as in specific subpopulations, including pregnant women and those living with chronic conditions and/or disabilities. These new 2020 Guidelines $^{12}$ and the methods used to identify the most current evidence underpinning them are described in detail in Bull et al. ${ }^{12}$

While reviewing the available evidence, a number of gaps in the existing literature were identified and the Guidelines ${ }^{12}$ highlighted the need for future research in several areas. ${ }^{13,14}$ Among those evidence gaps were that across the life course and for all different subgroups there is a common lack of information on: (1) the joint association between physical activity and sedentary time with health outcomes; and (2) the health benefits of light-intensity physical activity (LIPA). For example, there is limited information about how sedentary time modifies the beneficial health effects associated with physical activity and vice versa. ${ }^{15,16}$ Many people are constrained to remain sedentary for long periods of time daily, either because of their occupation, chronic conditions, or disabilities. Therefore, it is important to be able to quantify how levels of physical activity should be adapted for improved health at different levels of SB.

Indeed, although recommendations are made about the amount of moderate- to vigorous-intensity physical activity (MVPA) associated with health benefits, there are diverse opinions about 
recommendations on a specific threshold of sedentary time. The WHO and Physical Activity Guidelines for Americans, 2nd Edition did not set a threshold, but recently, 24-hour movement guidelines were issued for adults in $\mathrm{Canada}^{17}$ with a specific message to limit sedentary time to 8 hours or less.

The WHO Guidelines recommend increasing physical activity levels for those who have to spend long periods of time sedentary. However they did not provide specific information on how the amount of physical activity should be changed for different levels of sedentary time. The Physical Activity Guidelines for Americans, 2nd Edition contains a heat map that illustrates how the risk of allcause mortality changes with different combinations of time spent in SB and physical activity but does not provide quantitative information. ${ }^{10}$

This gap is in part due to the methodological and conceptual approaches used to date to build the evidence base underpinning physical activity recommendations. Previous research has tried to estimate dose-response associations between MVPA and SB independently; however, this is difficult to do because the day is limited to 24 hours, and therefore, these 2 behaviors are codependent, along with light-intensity activity (including standing) and sleep. Few studies have investigated the joint prospective association between SB and physical activity with health outcomes, such as all-cause mortality ${ }^{15,16,18,19}$; fewer still have accounted for the influence of sleep and LIPA. ${ }^{12,20}$

A possible alternative is to quantify the risk associated with combinations of time spent in these behaviors and to rethink recommendations in terms of the balance of time spent between physical activity and SB. In other words, we could seek to answer the question "What is the proportion of time during the day one should spend in MVPA for health benefits given the proportion of time spent in SB?"

In this paper, we present alternative ways to provide prospective evidence about the relationship between time spent in physical activity and SB and health outcomes, and examine how this could be translated into a combined recommendation in future guidelines. We illustrate this based on the associations of physical activity and SB with all-cause mortality using a publicly available data set.

\section{Methods}

\section{Data}

We used data from the 2005-2006 wave of the US National Health and Nutrition Examination Survey (NHANES), which used stratified, multistage probability sampling to recruit a sample representative of the civilian noninstitutionalized US population. Full details of the NHANES methodology can be found elsewhere. ${ }^{21}$ Mortality data linkage which provides vital status is available for a subset of participants. This records the length of time (in months) between the NHANES examination and a participant's death, up to December 2015.

\section{Measurement of the Time Spent in Physical Activity, SB, and Sleep}

Time spent in SB, LIPA, and MVPA was assessed using an ActiGraph accelerometer (AM-7164; ActiGraph, LLC, Fort Walton Beach, FL) following the protocol detailed previously. ${ }^{22}$ Briefly, participants were asked to wear the device on a belt around the waist for 7 consecutive days except when sleeping or bathing.
The resulting acceleration counts, integrated over 1-minute epochs, were processed according to the Centers for Disease Control and Prevention's standard quality assurance procedures. ${ }^{22}$ Daily accelerometry data were considered valid if the accelerometer was worn for at least 10 hours, and participants were included if they accumulated at least one valid day of activity as in previous studies. $^{23,24}$ The standard definition of nonwear time from the Centers for Disease Control and Prevention was adopted. This defines nonwear time as intervals of at least 60 consecutive minutes of $0 \mathrm{cpm}$ with allowances for up to 2 minutes of limited movement $(<50 \mathrm{cpm})$ within these periods. We classified time using standard count per minutes thresholds as SB ( $<100$ counts/min), LIPA (1002020 counts/min), or MVPA (>2020 counts/min). ${ }^{25}$ Sleep duration was self-reported to the nearest hour in response to the question "How much sleep do you actually get at night on weekdays or workdays?" Sleep time was then calculated as a proportion of 24 hours, and time spent in SB, LIPA, and MVPA was calculated as proportions of the remaining time (the waking day) according to the total time recorded for each behavior.

\section{Covariates}

We included as covariates variables representing the main known confounders in the relationships between daily activity and all-cause mortality including demographics, social economic status, other health behaviors, preexisting conditions, and physical limitations on movement. Sociodemographic covariates included age (in years); sex (male and female); race/ethnicity (Mexican American, other Hispanic, nonHispanic White, non-Hispanic Black, and other including mixed race); education (< ninth grade, 9-11th grade, high school, college or AA degree, college graduate, refuse to answer, and do not know); marital status (married, widowed, divorced, separated, never married, living with partner, refused, and don't know); and family income to poverty ratio (continuous from 0 to 4.99 , values are 5 if the ratio is 5 or over). Health behavior covariates included smoking status (yes, no, and former); average alcohol consumption (number of drinks per day over last $12 \mathrm{mo}$ ); average dietary intake (in kilocalories per day); average saturated fat intake (in grams per day); and average caffeine intake (in milligrams per day). Health status covariates included previous diagnosis of stroke (yes and no); previous diagnosis of cancer (yes and no); previous diagnosis of diabetes (yes and no); self-assessed health (poor, fair, good, very good, and excellent); use of medication to control blood pressure (yes and no); and physical limitations on movement (yes and no). All these covariates were measured via self-report as part of the interview.

\section{Statistical Analysis}

Model. The first step was to develop a statistical model that would allow us to estimate the mortality risk associated with any combination of time spent in physical activity and SB, while accounting for the time spent sleeping. This model should give more accurate estimates than the methods used by Powell et $\mathrm{al}^{11}$ based on interpolating between only 4 broad levels of MVPA and SB. ${ }^{26}$

We used a multivariate method based on the compositional approach developed by McGregor et $\mathrm{al}^{27}$ in which the day was defined as the proportions of time spent in $D=4$ movement behaviors: MVPA, LIPA, SB, and sleep (Sleep). In order to use the whole composition in a Cox regression model to estimate prospective risk of mortality the times spent in sleep, SB, LIPA, and MVPA were transformed into 3 isometric log-ratio coordinates, ${ }^{28}$ given by Equations $1-3$ : 


$$
\begin{gathered}
z_{1}=\sqrt{\frac{3}{4}} \ln \frac{\text { Sleep }}{\sqrt[3]{\mathrm{MVPA} \times \mathrm{LIPA} \times \mathrm{SB}}} \\
z_{2}=\sqrt{\frac{2}{3}} \ln \frac{\mathrm{LIPA}}{\sqrt{\mathrm{MVPA} \times \mathrm{SB}}} \\
z_{3}=\sqrt{\frac{1}{2}} \ln \frac{\mathrm{MVPA}}{\mathrm{SB}}
\end{gathered}
$$

The first coordinate $z_{1}$ represents time spent in sleep relative to the (geometric) average of all the other behaviors. The second coordinate $z_{2}$ is the balance between time spent in LIPA and the geometric average of time spent in MVPA and SB. The third coordinate $z_{3}$ accounts for the balance of time between moderate- to vigorous-intensity activity and $\mathrm{SB}$.

These coordinates (the vectors $\left.z=\left(z_{1}, z_{2}, z_{3}\right)^{T}\right)$ were then used as explanatory variables together with a set of covariate $v$ to fit a Cox regression model of the form shown in Equation 4:

$$
\ln \frac{h(t ; \boldsymbol{w})}{h_{0}(t)}=\sum_{j=1}^{D-1} \gamma_{j} z_{j}+\boldsymbol{\beta}^{T} \boldsymbol{v}=\boldsymbol{\gamma}^{T} z+\boldsymbol{\beta}^{T} \boldsymbol{v},
$$

where the function $h_{0}(t)$, depending upon time $t$, is an unspecified baseline hazard function, and the vectors $\boldsymbol{\gamma}$ and $\boldsymbol{\beta}$ are the corresponding regression coefficients. These coefficients were fitted in the usual manner by maximizing the partial likelihood function. ${ }^{27}$ The estimated associations between the previous time balances, as represented by the isometric log-ratio coordinates, and mortality were expressed as hazard ratios (HRs) with their corresponding $95 \%$ confidence intervals (CIs). ${ }^{27}$ Our modeling was restricted to adults between ages 50 and 79 years in line with previous work done on NHANES data to prevent violations of the proportional hazards assumption, ${ }^{29}$ which we verified using Kaplan-Meier curves and a Grambsch-Therneau test. ${ }^{30}$

\section{Calculation of Heat Map}

We plotted HRs estimated by the model as a heat map for combinations of time spent in MVPA and SB for a fixed amount of sleep time (fixing the duration of the waking day) as in McGregor et al. ${ }^{31}$ We restricted the range of values to plausible behavioral patterns. We computed the distribution of composition within the sample and considered only compositions within 2 SDs of the mean composition. Similarly, to choose a reference composition in the computation of HRs, we selected a point on the $75 \%$ confidence region for distribution, computed using Mahalanobis distance (a multivariate distance accounting for the covariance structure) ${ }^{32}$ that represented low levels of physical activity and high sedentary time $(M V P A=2 \mathrm{~min} / \mathrm{d}, \mathrm{LIPA}=229.0 \mathrm{~min} / \mathrm{d}, \mathrm{SB}=$ $729.0 \mathrm{~min} / \mathrm{d}$, and sleep $=480 \mathrm{~min} / \mathrm{d}$ ).

\section{Dose-Response of the Balance of Time Spent in MVPA and SB With Risk of Mortality}

The coordinate $z_{3}$ allows us to estimate directly the dose-response association between the ratio of time spent in MVPA and SB with risk of mortality. Given a fixed duration of the waking day (fixed sleep time), we also estimated the combinations of time spent in MVPA and SB that correspond to mortality risk level that can be achieved by being active according to the Physical Activity
Guidelines for Americans, 2nd Edition. ${ }^{10}$ Statistical models were adjusted for the covariates listed previously and associations reported as HR with $95 \%$ CIs. Sensitivity analysis excluding deaths within the first 2 years of follow-up was conducted to minimize the risk of reverse causality bias.

All statistical analyses and graphical representations were performed using the $\mathrm{R}$ system for statistical computing ( $\mathrm{R}$ version 3.4.1; R Foundation for Statistical Computing, Vienna, Austria, 2017). Statistical test significance was concluded at the usual .05 significance level. All codes are available as open source on https:// opencoda.net/.

\section{Results}

\section{Sample}

A total of 10,348 adults participated in the NHANES 2005-2006 cycle. Among those, $\mathrm{n}=5560$ adults were followed-up with $\mathrm{n}=$ 1820 falling within the age range (50-79 y) considered in this analysis. A subsample of $n=1594$ individuals with valid accelerometry data, full set of covariates, and nonaccidental death records were included in this analysis. The data flow is presented in Figure 1.

Table 1 provides the characteristics of this analytical sample including the summary statistics for the daily time composition in terms of time spent in sleep, SB, LIPA, and MVPA. In this sample, $86 \%$ of the variance in daily time composition is attributable to time spent in MVPA, $9 \%$ to LIPA, and the rest to time spent in sleep and SB. Valid deaths are nonaccidental deaths.

\section{Associations With Mortality}

The compositional Cox model showed that the whole composition of daily time spent in sleep, SB, LIPA, and MVPA was significantly associated with mortality risk (Likelihood ratio test $P<$ $.001)$. Figure 2 shows the association HR for time spent in MVPA ranging from 1 to 90 minutes per day and time spent in SB ranging from 3 to 14 hours per day. Different combinations of time spent in MVPA, LIPA, and SB were associated with similar risk levels. For example, both point (a) $(\mathrm{MVPA}=13 \mathrm{~min}, \mathrm{LIPA}=7.5 \mathrm{~h}$,

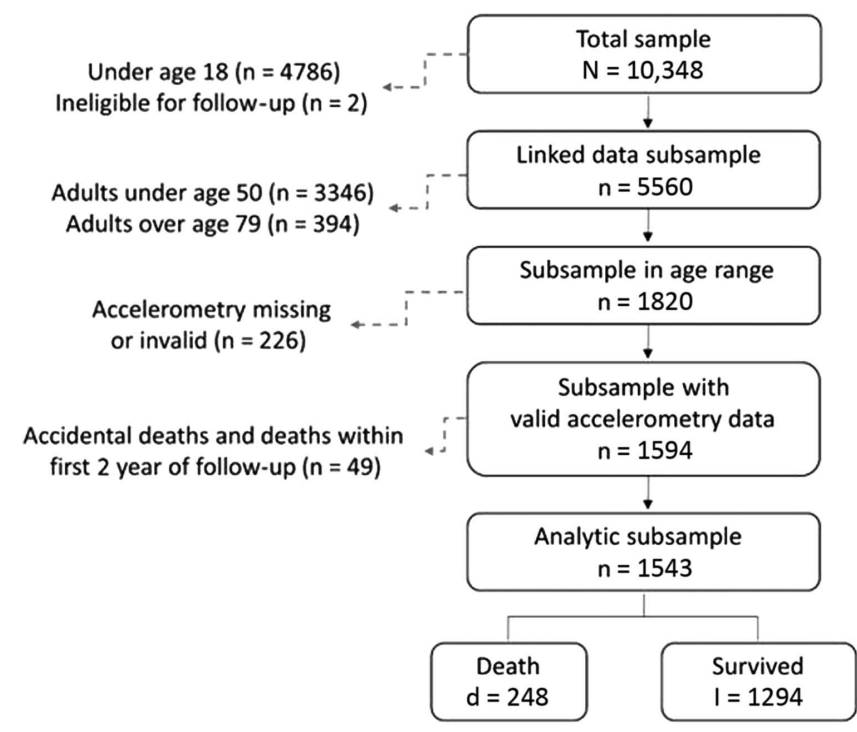

Figure 1 - The CONsolidated Standards of Reporting Trials diagram representing the data flow in this analysis. 
Table 1 Summary Statistics for the Final Analysis Sample

\begin{tabular}{|c|c|c|}
\hline Categorical covariates & Category & Proportion in sample, \% \\
\hline \multirow[t]{2}{*}{ Sex } & Male & 51.7 \\
\hline & Female & 48.3 \\
\hline \multirow[t]{4}{*}{ Smoking } & Current & 19.5 \\
\hline & Former & 35.6 \\
\hline & Never & 44.8 \\
\hline & Unknown & 0.1 \\
\hline \multirow[t]{6}{*}{ Self-rated health } & Excellent & 8.1 \\
\hline & Very good & 26.5 \\
\hline & Good & 35.0 \\
\hline & Fair & 21.4 \\
\hline & Poor & 4.1 \\
\hline & Unknown & 4.9 \\
\hline \multirow[t]{2}{*}{ Movement limitations } & Yes & 21.2 \\
\hline & No & 78.8 \\
\hline Continuous covariates & & Mean (SE) \\
\hline Age at baseline & & $63.1(0.2)$ \\
\hline Alcohol consumption, drinks/d & & $1.8(0.1)$ \\
\hline Mean energy intake, kcal/d & & $1940(21)$ \\
\hline Time composition, h/d & & Compositional mean \\
\hline Sleep & & 7.0 \\
\hline SB & & 10.5 \\
\hline LIPA & & 6.3 \\
\hline MVPA & & 0.2 \\
\hline
\end{tabular}

$\mathrm{SB}=8.3 \mathrm{~h}$ ) and (b) (MVPA $=26 \mathrm{~min}, \mathrm{LIPA}=5 \mathrm{~h}, \mathrm{SB}=10.5 \mathrm{~h})$ were associated with $\mathrm{HR}=0.50$. Similarly point (c) $(\mathrm{MVPA}=$ $30 \mathrm{~min}, \mathrm{LIPA}=7.2 \mathrm{~h}, \mathrm{SB}=8.3 \mathrm{~h})$ and $(\mathrm{d})(\mathrm{MVPA}=75 \mathrm{~min}$, $\mathrm{LIPA}=4.2 \mathrm{~h}, \mathrm{SB}=10.5 \mathrm{~h}$ ) were associated with $\mathrm{HR}=0.30$. Generally, risk of mortality was lower for less time spent in SB, corresponding also to higher time spent in LIPA. However, this was more pronounced at low levels of MVPA. At higher levels of daily MVPA, the effect of sedentary time appears attenuated. Displacing SB with MVPA required less time than displacing SB with LIPA for the same mortality rate, as indicated by the dashed lines with arrow on Figure 2. For example, moving from a HR of 0.5 to 0.3 from point (a) would require a change in composition of 50 minutes replacing SB but only a few minutes of MVPA.

Figure 3 shows the dose-response curve linking the balance of time spent in MVPA (in hours per day) and sedentary time (in hours per day) with risk of mortality. We observed a curvilinear relationship with decreasing risk of mortality for a higher amount of time spent in MVPA per hour of daily sedentary time. This curve simplifies the heat map presented in Figure 2 while retaining all the same information. It denotes the amount of daily MVPA required for a specific risk level for a given amount of daily sedentary time. A ratio of 0.017 (point [a]; Figure 3), which corresponds to 1 minute of MVPA per hour of daily sedentary time, was associated with a $\mathrm{HR}=0.63$ (95\% CI, 0.5-0.81). Hence for 8 hours of daily sedentary time, 8 minutes of MVPA, and 8 hours of light-intensity activity would be required to achieve this risk level (given $8 \mathrm{~h}$ of sleep as per reference point). To achieve the same HR, an individual remaining sedentary for 11 hours would require 11 minutes of MVPA and 5 hours of LIPA (given $8 \mathrm{~h}$ of sleep as per reference point). A ratio of 0.17 (point [c]; Figure 3), which corresponds to 10 minutes of MVPA per hour of daily sedentary time is associated with $\mathrm{HR}=0.31$ (95\% CI, 0.23-0.40). Around the inflection point, which is often used as a cutoff point to provide the lower end of physical activity recommendations, as it corresponds to a point at which most of the benefits have been realized, ${ }^{10}$ the balance of time is about 2.5 minutes of moderate to vigorous activity per hour of daily sedentary time (point [b]; Figure 3 , ratio $=0.04$ ). The possible combination of ways to achieve a specific level of mortality risk reduction can be represented graphically as in Figure 4. This provides the amount of daily MVPA and LIPA with $95 \%$ CI for a given amount of daily sedentary time associated with around $30 \%$ mortality risk reduction.

\section{Discussion}

Our analysis highlights a novel method to produce evidence about the health risk/benefits (here for all-cause mortality risk) associated with combinations of time spent in different classes of physical behavior, including physical activity and SB. Generally, our results are consistent with previous research produced using standard regression-based methods. ${ }^{10,11,15}$ We show that there is a doseresponse relationship between the balance of daily time spent in MVPA and SB with risk of all-cause mortality. We estimated this using a robust methodology that takes into account daily time spent 

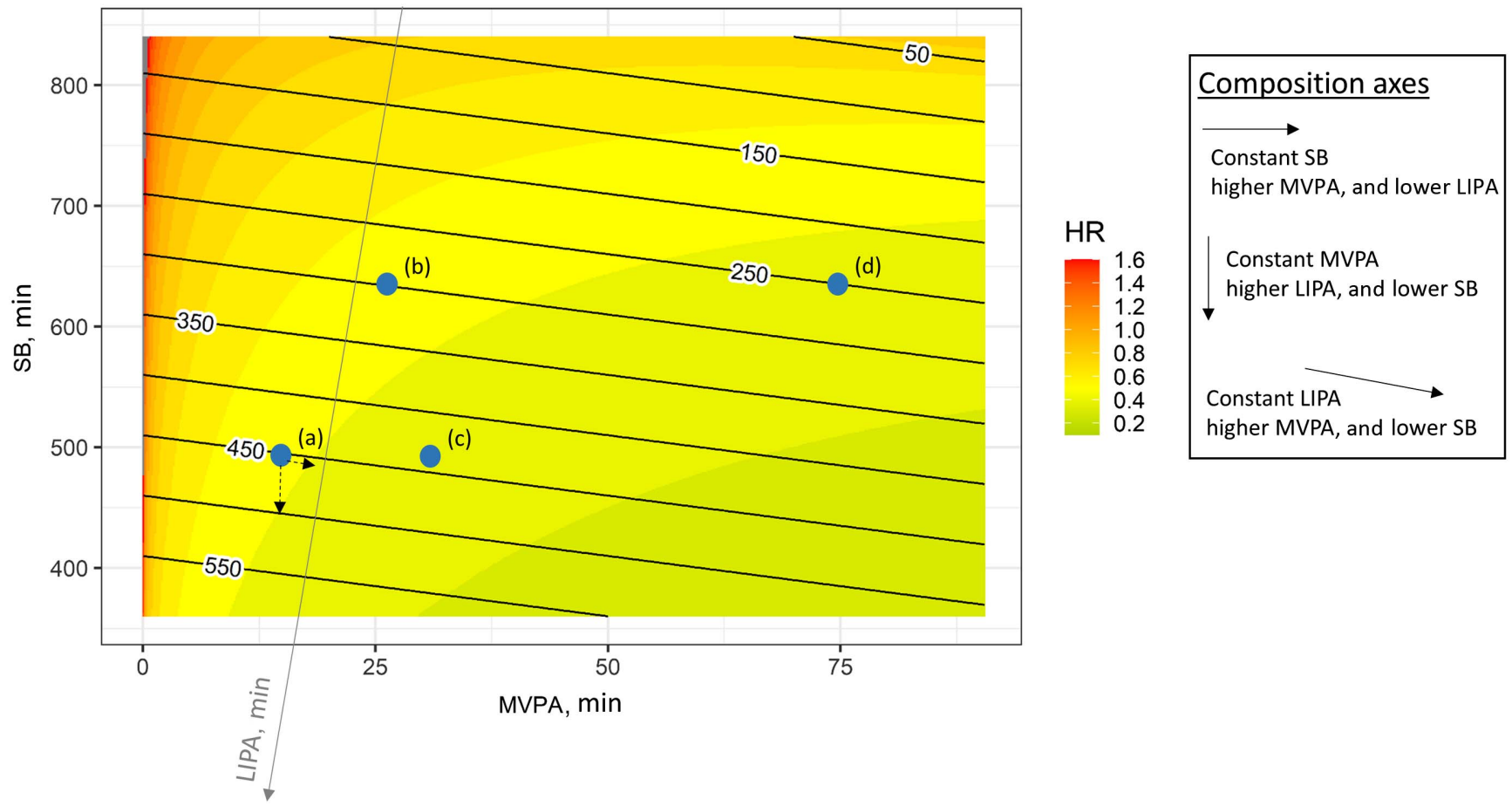

Figure 2 - Relationship between risk of all-cause mortality (color) with the daily composition of time spent in daily in MVPA ( $x$-axis), SB ( $y$-axis), and LIPA (contour lines gray axis). HR indicates hazard ratios; LIPA, light-intensity physical activity; MVPA, moderate- to vigorous-intensity physical activity; SB, sedentary behavior.

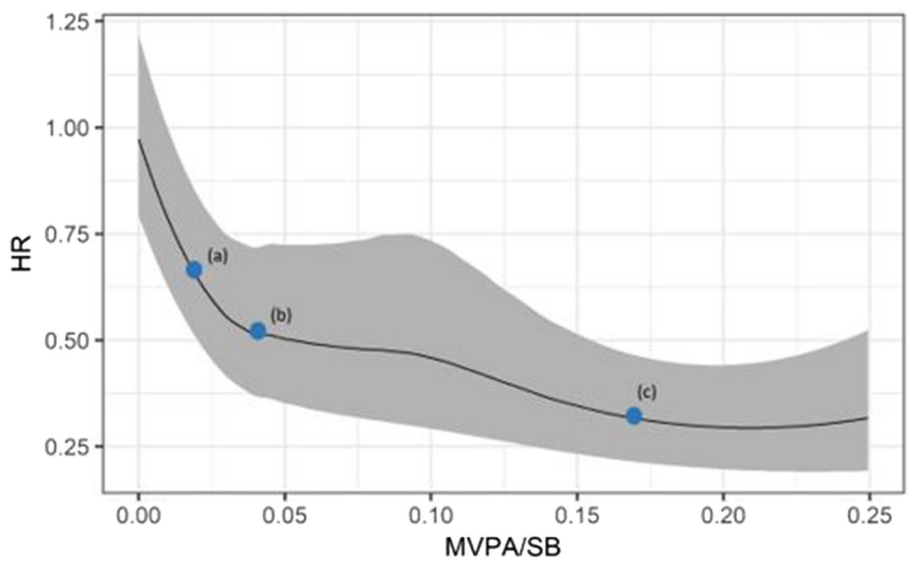

Figure 3 - Dose-response relationship between the balance of daily hours spent in MVPA and SB with risk of mortality. Point (a) corresponds to 1 minute of MVPA for every hour of SB. Point (b) corresponds to 2.5 minute of MVPA for every hour of SB. Point (c) corresponds to 10 minute of MVPA for every hour of SB. HR indicates hazard ratios; MVPA, moderate- to vigorous-intensity physical activity; SB, sedentary behavior.

in LIPA and sleep. This is another possible direction for future research to help addressing knowledge gaps about the interaction between physical activity and SB using robust statistical methods. This could be applied to other prospective health outcomes beyond all-cause mortality. Our approach could also be expanded to take into account more fine-grained energy expenditure and posture classes. This could be particularly useful for investigating, for example, if time spent in vigorous intensity per time unit activity is more efficient for improving health. ${ }^{33}$
We show that similar reductions in risk of all-cause mortality are associated with different combinations of time spent in MVPA, LIPA, and SB. Our analyses provide the first quantitative estimates of the heat map developed by the US Department of Health and Human Services Physical Activity Guidelines Advisory Committee ${ }^{10,11}$ based on a single data set (instead of interpolations) and adjusted for sleep time. Producing evidence on different combinations of physical activity and SB associated with the same health benefits could open the door to more flexible recommendations to suit an individual's circumstances and abilities.

Considering that sedentary time is often constrained by occupation, environment, or physical capacity over which individuals have less control, the question remains whether physical activity recommendations could be adapted to fit different circumstances and contexts. Thinking in terms of the "balance" of time could allow for better integration of recommendations on physical activity and SB. ${ }^{34}$ For example, based on Figure 4 for individuals who are sedentary for a long time $(11 \mathrm{~h}), 40$ minutes of daily moderate to vigorous physical activity would be required for a $30 \%$ risk reduction for all-cause mortality. Alternatively, for less sedentary individuals ( $6 \mathrm{~h}$ ), only 5 minutes might be sufficient to obtain the same risk reduction. As time spent in physical activity, $\mathrm{SB}$, and sleep are codependent, adopting a balance-of-time balanced approach to integrate guidelines for different behavior (eg, MVPA and SB) might be more relevant and accurate when compared with combining recommendations about individual behaviors based on evidence derived from individual behaviors. ${ }^{4}$ This type of evidence could pave the way to more integrated guidelines based on balance of activity behaviors, such as "Aim for $x$ minutes of MVPA for every $x$ hours of the day you usually sit" or presented in visual form like the heat map in Figure 2 and American Physical Activity Guidelines, ${ }^{10,11}$ and potentially inform 24-hour movement guidelines. ${ }^{17}$ 


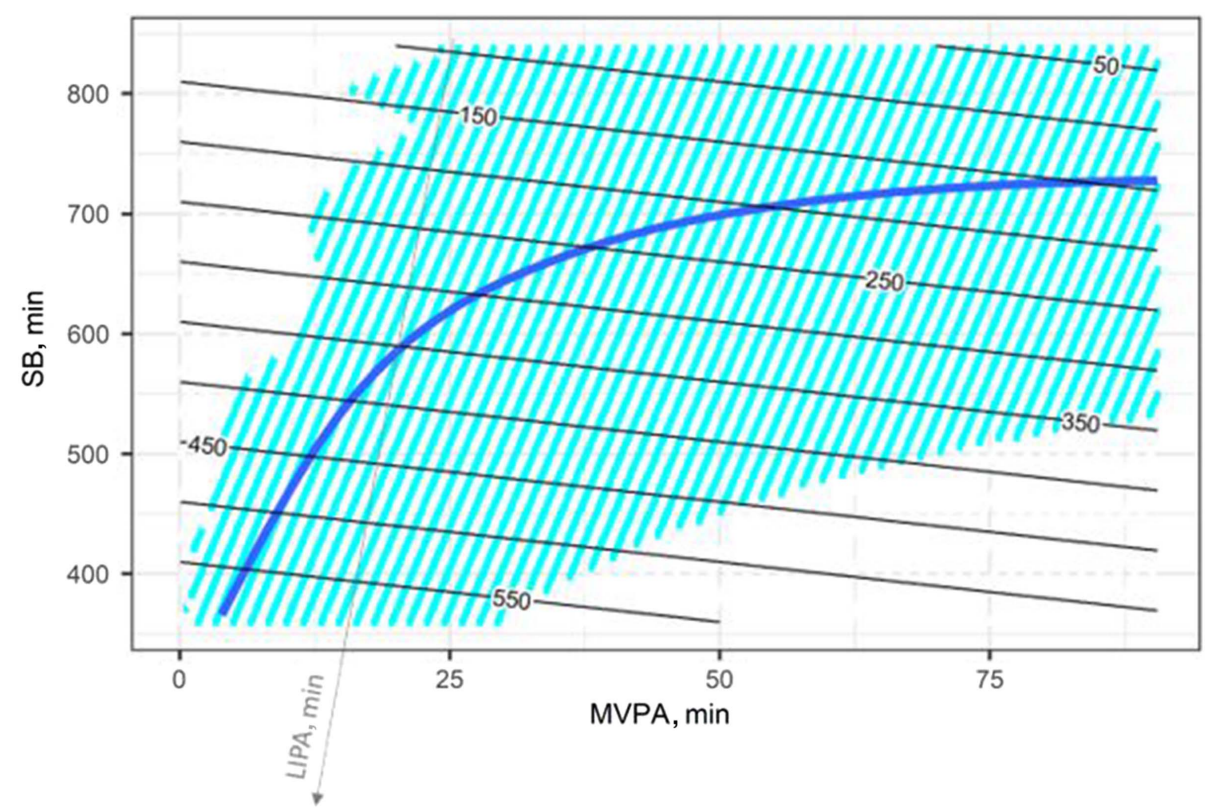

Figure 4 - Amount of MVPA ( $x$-axis) per time spent in SB ( $y$-axis) and LIPA (oblique lines) associated with around $30 \%$ mortality risk reduction. Corresponding time spent in LIPA for each pair of time spent in MVPA and SB is indicated by contour lines (gray axis). Blue hash lines indicate the 95\% CI. CI indicates confidence interval; LIPA, light-intensity physical activity; MVPA, moderate- to vigorous-intensity physical activity; SB, sedentary behavior.

\section{Strengths and Limitations}

While this study provides potential methodological pathways to improve integration between activity behaviors, important evidence gaps include the availability and access to quality individual participant data. Recently, the possibility of deriving meaningful prospective information from some major cohorts has been questioned because of difficulties in measuring sedentary and standing time, short follow-up time, and issues with timing of measurement of different covariates. ${ }^{35,36}$ However, there are a number of initiatives, such as PROPASS, aimed at consolidating cohort data resources, ${ }^{37}$ which might provide solutions. Harmonized and federated analysis methods are also helping to improve the situation. ${ }^{15,38,39}$

The quantitative results in this report need to be interpreted with caution, and they do not constitute recommendations as there are a number of limitations associated with this study. This is a small sample illustrative study. Waist-worn accelerometers have limitations for quantifying sedentary time as they cannot differentiate between sitting and standing with standing time often misclassified as sedentary time. ${ }^{37}$ Besides, they are taken off during sleep times, and for this reason, we relied on sleep self-reports in this study that may be overestimated. In addition, there is the possibility that unmeasured confounding factors might explain some of the dose-response associations we observed. Finally, the possibility of reverse causality cannot be completely eliminated. Our results reflect the physical activity and SB patterns of the US population other compositional data analysis studies on different health outcomes, in ethnically diverse samples, including studies from low and middle income countries, will be crucial for future updates of the global PA and SB guidelines.

\section{Conclusion}

Quantifying the dose-response between the balance of time spent in moderate to vigorous activity and sedentary time with health indicators using a compositional data analysis approach can provide evidence for future integrated recommendations that reflects the interactions between different physical behaviors, such as MVPA and SB. Several combinations of time spent in these behaviors are associated with similar risk levels; this opens the door to more flexible recommendations for future guidelines.

\section{References}

1. World Health Organization. Guidelines on Physical Activity and Sedentary Behaviour. Author; 2020.

2. World Health Organization. Guidelines on Physical Activity, Sedentary Behaviour and Sleep for Children Under 5 Years of Age. International Government Publication: Author; 2019.

3. World Health Organization. Global Recommendations on Physical Activity for Health. Geneva: Author; 2010.

4. Tremblay MS, Carson V, Chaput J-P, et al. Canadian 24-hour movement guidelines for children and youth: an integration of physical activity, sedentary behaviour, and sleep. Appl Physiol Nutr Metab. 2016;41(6, suppl 3):S197-S239. doi:10.1139/apnm-2016-0151

5. Carson V, Hunter S, Kuzik N, et al. Systematic review of sedentary behaviour and health indicators in school-aged children and youth: an update. Appl Physiol Nutr Metab. 2016;41(6, suppl 3):S240-S265. PubMed ID: 27306432 doi:10.1139/apnm-2015-0630

6. Poitras VJ, Gray CE, Borghese MM, et al. Systematic review of the relationships between objectively measured physical activity and health indicators in school-aged children and youth. Appl Physiol Nutr Metab. 2016;41:S197-S239.

7. Australian Government, Department of Health. Australia's physical activity and sedentary behaviour guidelines and the Australian 24-hour movement guidelines [published online ahead of print 2019]. Educ Prev.

8. Mottola MF, Davenport MH, Ruchat SM, et al. 2019 Canadian guideline for physical activity throughout pregnancy. Br J Sports Med. 2018;52:1339-1346. 
9. Piercy KL, Troiano RP, Ballard RM, et al. The physical activity guidelines for Americans. JAMA. 2018;320(19):2020-2028. PubMed ID: 30418471 doi:10.1001/jama.2018.14854

10. US Department of Health and Human Services. 2018 Physical Activity Guidelines Advisory Committee Scientific Report; 2018.

11. Powell KE, King AC, Buchner DM, et al. The scientific foundation for the physical activity guidelines for Americans, 2nd edition. J Phys Act Health. 2019;16:1-11.

12. Bull F, Saad Al-Ansari S, Biddle S, et al. World Health Organization 2020 Guidelines on physical activity and sedentary behavior. $\mathrm{Br} J$ Sports Med. 2020;54(24), 1451-1462.

13. DiPietro L, Al-Ansari S, Biddle S, et al. Advancing the global physical activity agenda: Recommendations for future research by the 2020 WHO physical activity and sedentary behavior guidelines development group. Int J Behav Nutr Phys Act. 2020;17:143.

14. Dempsey P, Biddle S, Buman M, et al. New global guidelines on sedentary behaviour and health for adults: Broadening the behavioural targets. Int J Behav Nutri Phys Act. 2020;17:151.

15. Ekelund U, Steene-Johannessen J, Brown WJ, et al. Does physical activity attenuate, or even eliminate, the detrimental association of sitting time with mortality? A harmonised meta-analysis of data from more than 1 million men and women. Lancet. 2016;388(10051): 1302-1310. PubMed ID: 27475271 doi:10.1016/S0140-6736(16) 30370-1

16. Stamatakis E, Gale J, Bauman A, Ekelund U, Hamer M, Ding D. Sitting time, physical activity, and risk of mortality in adults. $J$ Am Coll Cardiol. 2019;73:2062-2072.

17. Ross R, Chaput J-P, Giangregorio LM, et al. Canadian 24-hour movement guidelines for Adults aged 18-64 years and Adults aged 65 years or older: an integration of physical activity, sedentary behaviour, and sleep. Appl Physiol Nutr Metab. 2020;45(10):S57S102. doi:10.1139/apnm-2020-0467

18. McGregor DE, Palarea-Albaladejo J, Dall PM, del Pozo Cruz B, Chastin SFM. Compositional analysis of the association between mortality and 24-hour movement behaviour from NHANES [published online ahead of print 2019]. Eur J Prev Cardiol.

19. von Rosen P, Dohrn I-M, Hagströmer M. Association between physical activity and all-cause mortality: a 15-year follow-up using a compositional data analysis. Scand J Med Sci Sports. 2020; 30(1):100-107. PubMed ID: 31581345

20. Janssen I, Clarke AE, Carson V, et al. A systematic review of compositional data analysis studies examining associations between sleep, sedentary behaviour, and physical activity with health outcomes in adults. Appl Physiol Nutr Metab. 2020;45(10):S248-S257. doi:10.1139/apnm-2020-0160

21. US Department of Health and Human Services Centers for Disease Control and Prevention. National Health and Nutrition Examination Survey. http://www.cdc.gov/nchs/nhanes.htm.

22. Troiano RP, Berrigan D, Dodd KW, Mâsse LC, Tilert T, McDowell M. Physical activity in the United States measured by accelerometer. Med Sci Sports Exerc. 2008;40(1):181-188. PubMed ID: 18091006 doi:10.1249/mss.0b013e31815a51b3

23. Healy GN, Dunstan DW, Salmon J, et al. Objectively measured lightintensity physical activity is independently associated with 2-h plasma glucose. Diabetes Care. 2007;30(6):1384-1389. PubMed ID: 17473059 doi:10.2337/dc07-0114
24. Schmid D, Ricci C, Leitzmann MF. Associations of objectively assessed physical activity and sedentary time with all-cause mortality in US adults: the NHANES study. PLoS One. 2015;10(3):e0119591. PubMed ID: 25768112 doi:10.1371/journal.pone.0119591

25. Freedson PS, Melanson E, Sirard J. Calibration of the computer science and applications, Inc. accelerometer. Med Sci Sports Exerc. 1998;30(5):777-781. PubMed ID: 9588623 doi:10.1097/00005768199805000-00021

26. Aitchinson J. The Statistical Analysis of Compositional Data. Blackburn Press; 2003.

27. McGregor D, Palarea-Albaladejo J, Dall P, Hron K, Chastin S. Cox regression survival analysis with compositional covariates: application to modelling mortality risk from 24-h physical activity patterns. Stat Methods Med Res. 2020;29(5):1447-1465. PubMed ID: 31342855 doi:10.1177/0962280219864125

28. Egozcue JJ, Pawlowsky-Glahn V. Groups of parts and their balances in compositional data analysis. Math Geol. 2005;37(7):795-828. doi:10.1007/s11004-005-7381-9

29. Fishman EI, Steeves JA, Zipunnikov V, et al. Association between objectively measured physical activity and mortality in NHANES. Med Sci Sports Exerc. 2016;48(7):1303-1311. doi:10.1249/MSS. 0000000000000885

30. Grambsch P, Therneau T. Proportional hazards tests and diagnostics based on weighted residuals. Biometrika. 1994;81(3):515-526. doi:10.1093/biomet/81.3.515

31. McGregor DE, Palarea-Albaladejo J, Dall PM, Stamatakis E, Chastin SFM. Differences in physical activity time-use composition associated with cardiometabolic risks. Prev Med Reports. 2019;13:23-29. doi:10.1016/j.pmedr.2018.11.006

32. Mahalanobis P. On the generalised distance in statistics. Proc Natl Inst Sci India. 1936;2(1):49-55.

33. Strain T, Wijndaele K, Dempsey PC, et al. Wearable-device-measured physical activity and future health risk. Nat Med. 2020;26:1385-1391.

34. Chaput J-P, Carson V, Gray CE, Tremblay MS. Importance of all movement behaviors in a 24 hour period for overall health. Int $J$ Environ Res Public Health. 2014;11(12):12575-12581. PubMed ID: 25485978 doi:10.3390/ijerph111212575

35. Strain T, Wijndaele K, Sharp SJ, Dempsey PC, Wareham N, Brage S. Impact of follow-up time and analytical approaches to account for reverse causality on the association between physical activity and health outcomes in UK Biobank. Int J Epidemiol. 2020;49:162-172.

36. Matthews CE, Troiano RP, Salerno EA, et al. Exploration of confounding due to poor health in an accelerometer-mortality study. Med Sci Sports Exerc. 2020;52:2546-2553.

37. Stamatakis E, Koster A, Hamer M, et al. Emerging collaborative research platforms for the next generation of physical activity, sleep and exercise medicine guidelines: the Prospective Physical Activity, Sitting, and Sleep consortium (ProPASS). Br J Sports Med. 2020; 54:435-437.

38. Ekelund U, Tarp J, Steene-Johannessen J, et al. Dose-response associations between accelerometry measured physical activity and sedentary time and all cause mortality: systematic review and harmonised meta-analysis. BMJ. 2019;366:14570. doi:10.1136/bmj.14570

39. Ekelund U, Dalene KE, Tarp J, Lee I-M. Physical activity and mortality: what is the dose-response and how big is the effect? $\mathrm{Br}$ J Sports Med. 2020;54:1125-1126. 